\section{BMJ Open} Ophthalmology

\title{
Are eyes the windows to COVID-19? Systematic review and meta-analysis
}

\author{
Rina La Distia Nora (D) , ${ }^{1,2}$ Ikhwanuliman Putera, ${ }^{1}$ Dhiya Farah Khalisha, \\ Indah Septiana, ${ }^{1}$ Asri Salima Ridwan, ${ }^{1}$ Ratna Sitompul ${ }^{1}$
}

To cite: La Distia Nora R, Putera I, Khalisha DF, et al. Are eyes the windows to COVID-19? Systematic review and meta-analysis. BMJ Open Ophthalmology 2020;5:e000563. doi:10.1136/ bmjophth-2020-000563

- Additional material is published online only. To view, please visit the journal online (http://dx.doi.org/10.1136/ bmjophth-2020-000563).

Received 8 July 2020 Revised 10 August 2020 Accepted 29 August 2020
Check for updates

\section{(C) Author(s) (or their} employer(s)) 2020. Re-use permitted under CC BY-NC. No commercial re-use. See rights and permissions. Published by BMJ.

${ }^{1}$ Department of Ophthalmology, Faculty of Medicine, Universitas Indonesia - Cipto Mangunkusumo Kirana Eye Hospital, Jakarta, Indonesia 2Department of Immunology, Erasmus Medical Center, Rotterdam, Netherlands

Correspondence to Dr Rina La Distia Nora; rina. ladistia@ui.ac.id

\section{ABSTRACT}

Objective To review and critically appraise the ocular manifestation and the presence of SARS-CoV-2 through PCR positivity from ocular samples in COVID-19-related patients. Moreover, to evaluate the time and severity association of ocular manifestation to systemic disease of COVID-19.

Methods and analysis A systematic literature search from PubMed, ScienceDirect and Google Scholar databases was performed using standardised Preferred Reporting Items for Systematic Reviews and MetaAnalyses guideline. Selected keywords were related to COVID-19, ocular manifestation and PCR testing of SARS-CoV-2. Studies were assessed for their validity, and the data were extracted by two independent reviewers. Observational, case series and case report studies were included if they met the selection criteria. Meta-analysis was performed to estimate the pooled prevalence of ocular manifestations and PCR positivity from tears.

Results Thirty-one articles were qualitatively reviewed, and 14 studies were included in the meta-analysis. The pooled prevalence of ocular manifestation among COVID19-related patients was 0.05 (95\% $\mathrm{Cl} 0.02 \%$ to 0.08$)$. The overall PCR from tears samples positivity rate from COVID19-related patients presenting with ocular manifestation was 0.38 (95\% Cl $0.14 \%$ to 0.65$)$. Ocular manifestation could precede systemic manifestation in about $0.28(95 \%$ $\mathrm{Cl} 0.05 \%$ to 0.58 ) of COVID-19-related patients with ocular manifestations. Besides, ocular manifestation was not associated with a severe form of COVID-19.

Conclusion Although the overall number of ocular manifestation and SARS-CoV-2 PCR positivity rate from ocular samples was very low, around a quarter of COVID19-related patients with ocular manifestation presented their ocular manifestation earlier than the systemic manifestation regardless of the severity. Interestingly, SARS-CoV-2 PCR was positive from one-third of ocular samples, which could potentially be the source of infection to the respiratory tract and the environment, although the infectivity is yet to be determined.

\section{INTRODUCTION}

The 2019 novel coronavirus (2019-nCoV) or SARS-CoV-2, a single-stranded positive-sense RNA virus, belongs to the family of Coronaviridae. COVID-19, the disease caused by SARS-CoV-2 infection, can range from being asymptomatic to critically ill, leading to death. ${ }^{12}$ In December 2019, SARS-CoV-2 started to spread, and COVID-19 had become

\section{Key messages}

What is already known about this subject?

- SARS-CoV-2 can infect and replicate in the eyes through angiotensin receptor enzyme-2 receptor found in conjunctiva and cornea.

- Conjunctivitis among patients with COVID-19 had been reported.

- The nasolacrimal duct can transmit the virus from the eyes to the nasopharynx.

\section{What are the new findings?}

- The overall prevalence of ocular manifestation among patients with COVID- 19 is $5 \%$.

- Around a quarter of ocular manifestation could precede systemic manifestations among COVID-19related patients with ocular manifestation.

SARS-CoV-2 PCR was positive from one-third of ocular samples among COVID-19-related patients with ocular manifestation.

\section{How might these results change the focus of research or clinical practice? \\ - Conjunctivitis in patients suspected to have COVID-19 could potentially be the source of infection due to SARS-COV-2. \\ D Detecting SARS-CoV-2 from ocular samples is dif- ficult. Yet, ophthalmologists or general practitioners facing conjunctivitis in patients highly suspected to have COVID-19 or in areas with high transmission of COVID-19 should wear adequate personal protective equipment, including mask and goggles/face shield.}

a global pandemic. By 14 May 2020, it was estimated that more than four million people being infected and 294046 deaths worldwide were caused by COVID-19. ${ }^{3}$ WHO issued the first set of personal protective equipment guideline in March 2020 based on the previous experience in managing Middle Eastern respiratory syndrome coronavirus and SARS-CoV in 2004 . $^{4}$ In their recommendation, wearing goggles or face shield was included as a protection against SARS-CoV-2 transmission, even though there was still lack of published studies reporting eye infection caused by COVID-19 at that time. From the previous SARS-CoV experience, coronavirus could be found in tears, based on PCR 
positivity. ${ }^{5}$ Human-to-human aerosol transmission has been described mainly via the respiratory tract through droplets. ${ }^{12}$ However, the possibility of SARS-CoV-2 transmission through the ocular surface in the population is maybe often overlooked.

$\mathrm{Lu}$ et at ${ }^{6}$ warned that ocular manifestation may have appeared earlier than predicted. Their argument was based on the report of Guangfa Wang, a member of the national expert panel who inspected Wuhan and then got infected by SARS-CoV-2 at the beginning of the COVID-19 spread. Wang described that his red eyes had started several days before respiratory symptoms of COVID-19 appeared. As it may appear earlier, the ocular manifestation may be underreported. Guan $e t a l^{7}$ in their first report of a COVID-19 patient in China, found that ocular manifestation only contributed in around $1 \%$ of patients. Subsequently, Wu et $a l^{8}$ reported that ocular manifestation might be as high as $30 \%$ among patients with COVID-19. In comparison to lung and bronchial tissue cultures, the highest viral replication of SARS-CoV-2 at 48 hours was found in conjunctival tissue culture based on the recent ex vivo study. ${ }^{9}$ Moreover, SARS-CoV-2 PCR from tears can be still positive up to 3 weeks from the onset of systemic symptom even though the nasopharyngeal swab result is already negative. ${ }^{10}$ As SARS-CoV-2 replication in ocular surface may continue for a relatively long period and the virus can be transported to nasopharyngeal mucosa through the nasolacrimal duct, ${ }^{11}$ there is a possibility that ocular manifestation can affect systemic COVID-19. With the growing number of recently published studies, we performed a systematic review and meta-analysis to elaborate the possibility of the eye as an infection source for systemic COVID-19 by looking at the ocular manifestation and the presence of SARS-CoV-2 in the eye. We also aimed to describe the ocular clinical manifestation and the onset of ocular symptoms of COVID-19 with their relation to the presenting systemic manifestation along with its severity level.

\section{METHODS}

\section{Literature search strategy}

We performed a systematic review according to Preferred Reporting Items for Systematic Reviews and Meta-Analyses (PRISMA) guideline. ${ }^{12}$ Literature search was conducted from 4 to 9 June 2020 using three electronic databases: PubMed, ScienceDirect and Google Scholar. The literature search would be expanded using a snowballing method to the references of retrieved papers. Articles were identified with search strategy: "SARS-CoV-2 [supplementary concept]" OR "2019-nCoV" OR "COVID-19 [supplementary concept]" AND "conjunctiv*" OR "eye manifestations (MeSH)" OR "cornea (MeSH)" OR "ocular surface" OR "dry eye syndromes (MeSH)". This study was registered to the International Prospective Register of Systematic Reviews (PROSPERO registration number CRD42020194245).

\section{Eligibility criteria}

The enrolment date of studies was restricted from December 2019 to 1 June 2020. Records were managed by Mendeley software to exclude duplicates. Articles in English and human subjects were obtained. The inclusion criteria were (1) peer-reviewed observational case series and case report studies of COVID-19-related patients, including confirmed and suspected cases; (2) studies providing ocular manifestations; (3) if available, studies reporting PCR positivity in either ocular, elsewhere samples or both. Studies that reported ocular involvement but did not describe the ocular manifestations or reported only subjective ocular complaints were excluded. If it is necessary, the original author of each study was contacted by email to request further information.

\section{Data extraction}

Three authors (RLDN, IS and DFK) independently reviewed titles and abstracts generated by the search. A standardised data abstraction table was designed to capture all relevant information required for analysis. For all included studies, we recorded the following information: author, date of publication, study design, PCR positivity in nasal swab and tears, the onset of ocular manifestation, description of ocular and systemic manifestations, and disease severity. Ocular symptom duration and treatment given by the healthcare providers were also noted whenever the data are available.

\section{Quality assessment}

Two authors independently assessed the quality of included studies (DFK and ASR). The risk of bias and quality of primary studies or systematic reviews were assessed using the Newcastle-Ottawa Scale for longitudinal and cross-sectional studies. ${ }^{13}$ The quality levels then were graded as good, fair or poor. For case series and case report studies, the quality was assessed using Murad et $a l \mathrm{~s}^{14}$ set of criteria and graded as poor, moderate $\mathrm{r}$ good quality. Discrepancies and disagreements were resolved by consensus, and/or resolution of the conflict was performed by a third reviewer (IP) if necessary.

\section{Patient involvement}

Patients were not directly involved in the design of this study.

\section{Statistical analysis}

We undertook an initial descriptive analysis of the studies. The heterogeneity between estimates was assessed using the $\mathrm{I}^{2}$ statistic. For studies with calculable prevalence for each item, the meta-analysis was performed using a random-effects model conducted using the MetaXL 5.3 ( www.epigear.com) add-in for Microsoft Excel Professional Plus 2013. A pooled prevalence figure was calculated with a $95 \%$ CI. The pooled OR for the association of ocular manifestation with COVID-19 severity was calculated using a random model effect. The Mantel-Haenszel method was used to weight the studies. This statistical approach was performed using Review Manager V.5.4.

\section{RESULTS}

\section{Study selection}

Our initial search identified 31 studies to be analysed qualitatively in our systematic review. There were 5 


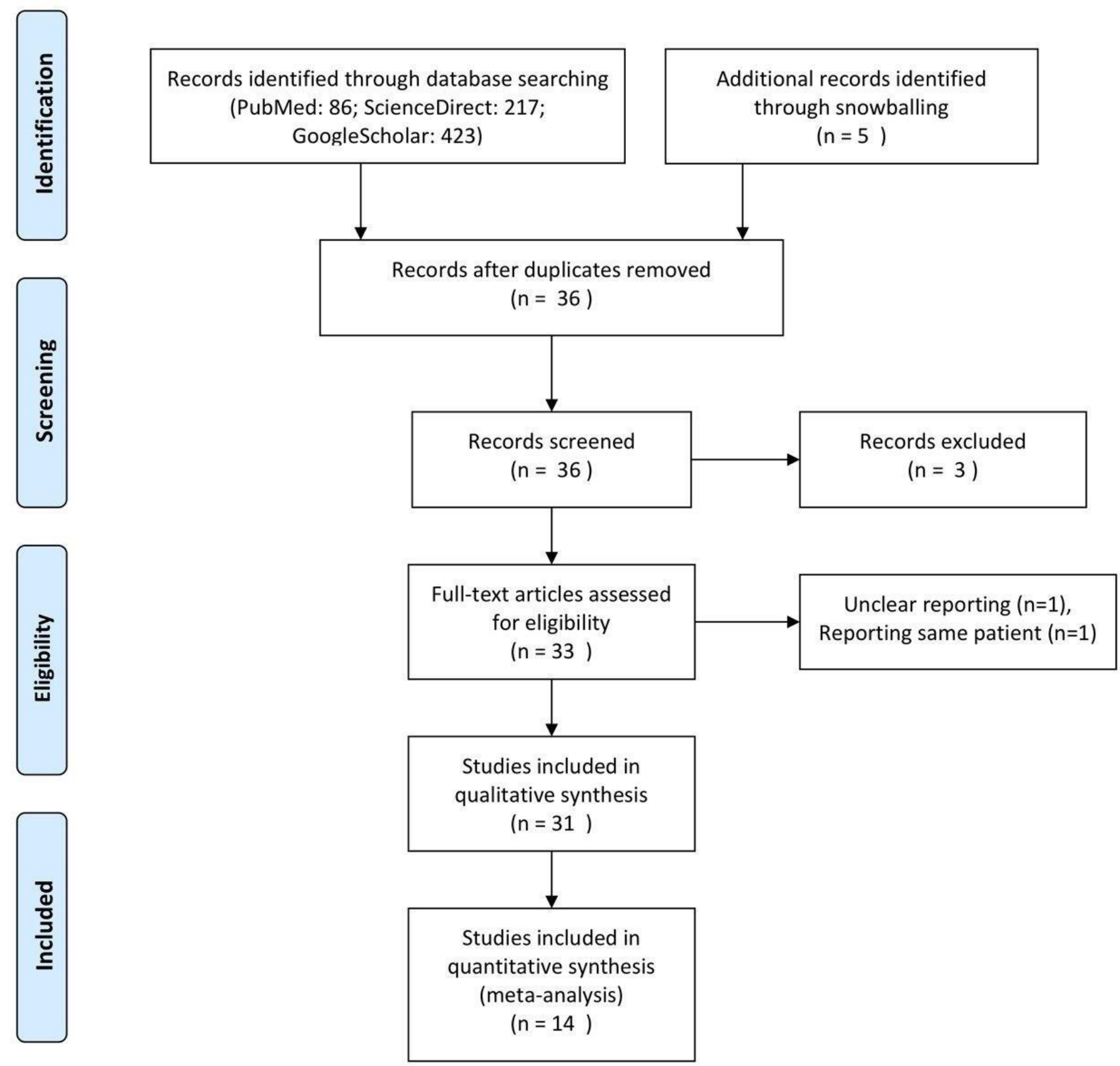

Figure 1 Preferred Reporting Items for Systematic Reviews and Meta-Analyses flowchart for the study selection process.

longitudinal, 9 cross-sectional, 5 case series, and 12 case report studies being reviewed for analysis. For quantitative analysis, 14 studies could be included in the pooled meta-analysis of prevalence. The flowchart of study selection is illustrated in figure 1. All articles were assessed for validity (online supplemental file). Tables 1 and 2 summarise the characteristics and main findings of the observational and case report studies, respectively.

Ocular manifestation and PCR positivity rate for SARS-CoV-2 among COVID-19-related patients

A meta-analysis to determine the estimation of ocular manifestation among COVID-19-related patients was performed. The pooled prevalence of ocular manifestation among COVID-19-related patients was 0.05 $(0.02 \%-0.0895 \% \mathrm{CI})$. The $\mathrm{I}^{2}$ for heterogeneity test was $86 \%$, suggesting a high level of heterogeneity (figure 2). Most of the studies with a large number of patients (Guan $e t a l^{7} \mathrm{Wu}$ et $a l^{8}$ and Zhang $e a^{1 l^{5}}$ ) included patients already admitted to the hospital. In addition, most of the studies included in this study reported only ocular complaints from their subjects without any further ocular examination.

From COVID-19-related patients with ocular manifestations, overall PCR positivity from tears samples was 0.38 ( $95 \%$ CI $0.14 \%$ to 0.65 , figure 3 ). The pooled PCR positivity from tears was higher compared with general PCR 


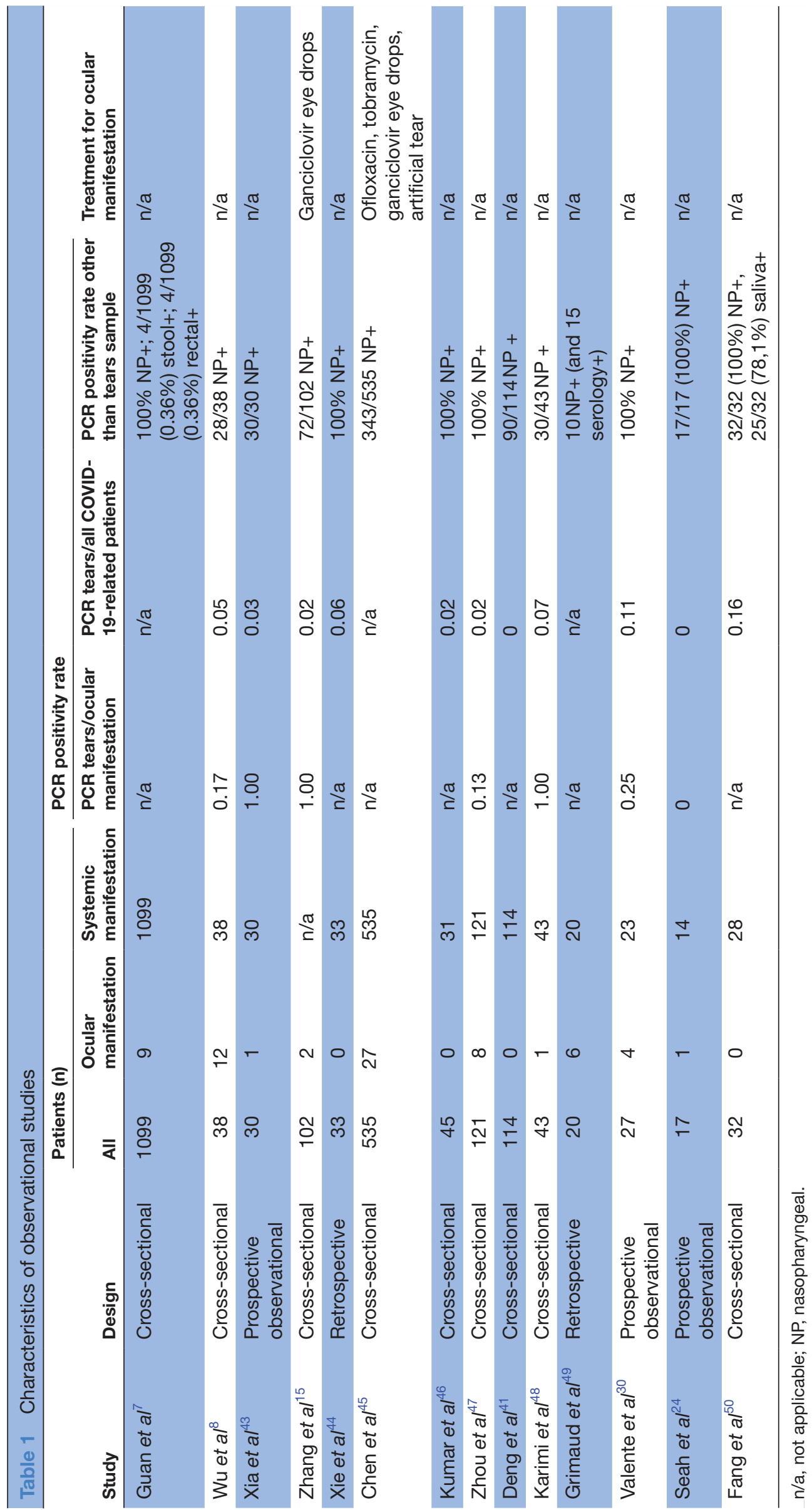

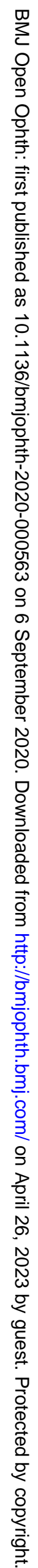




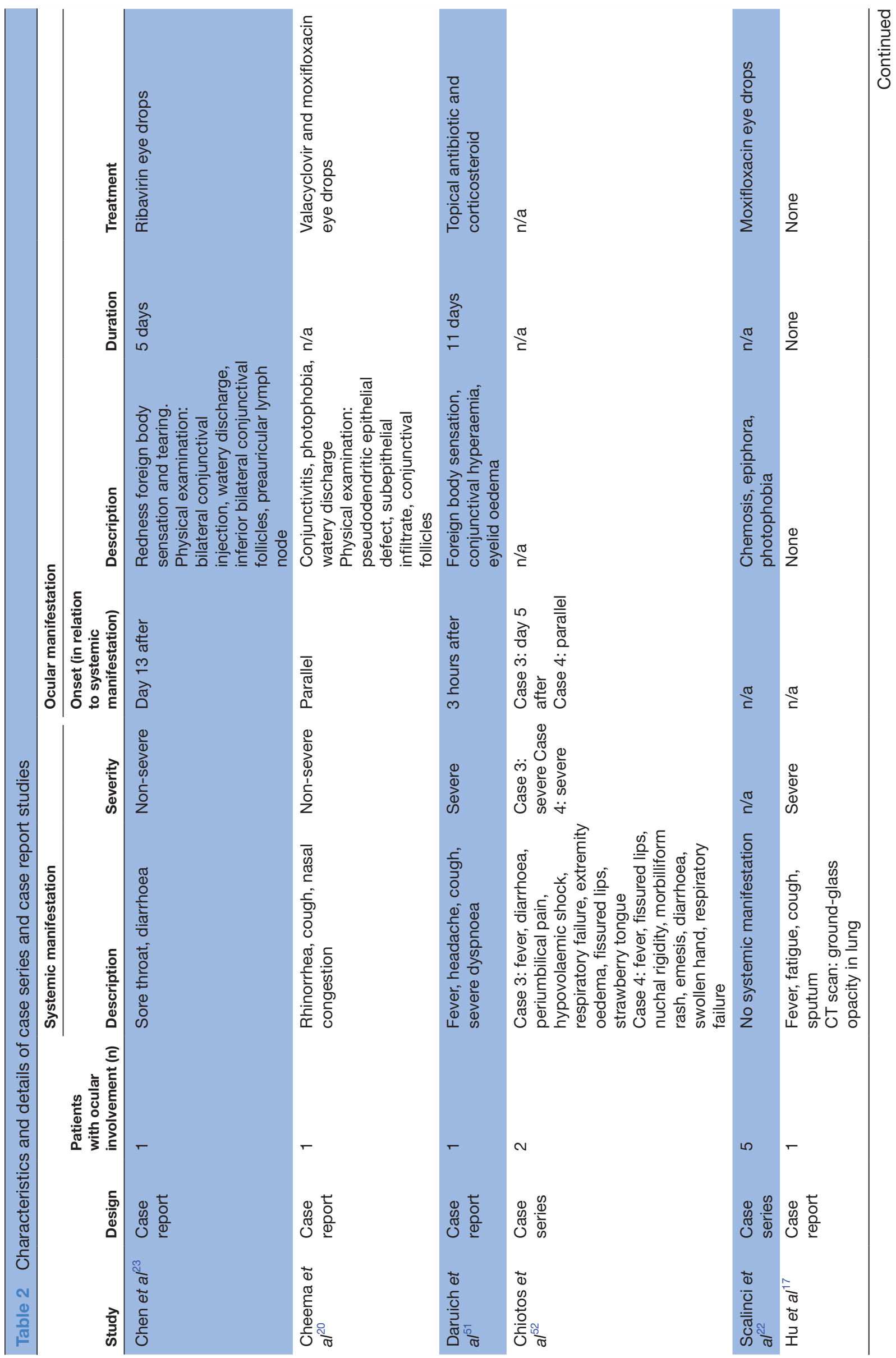




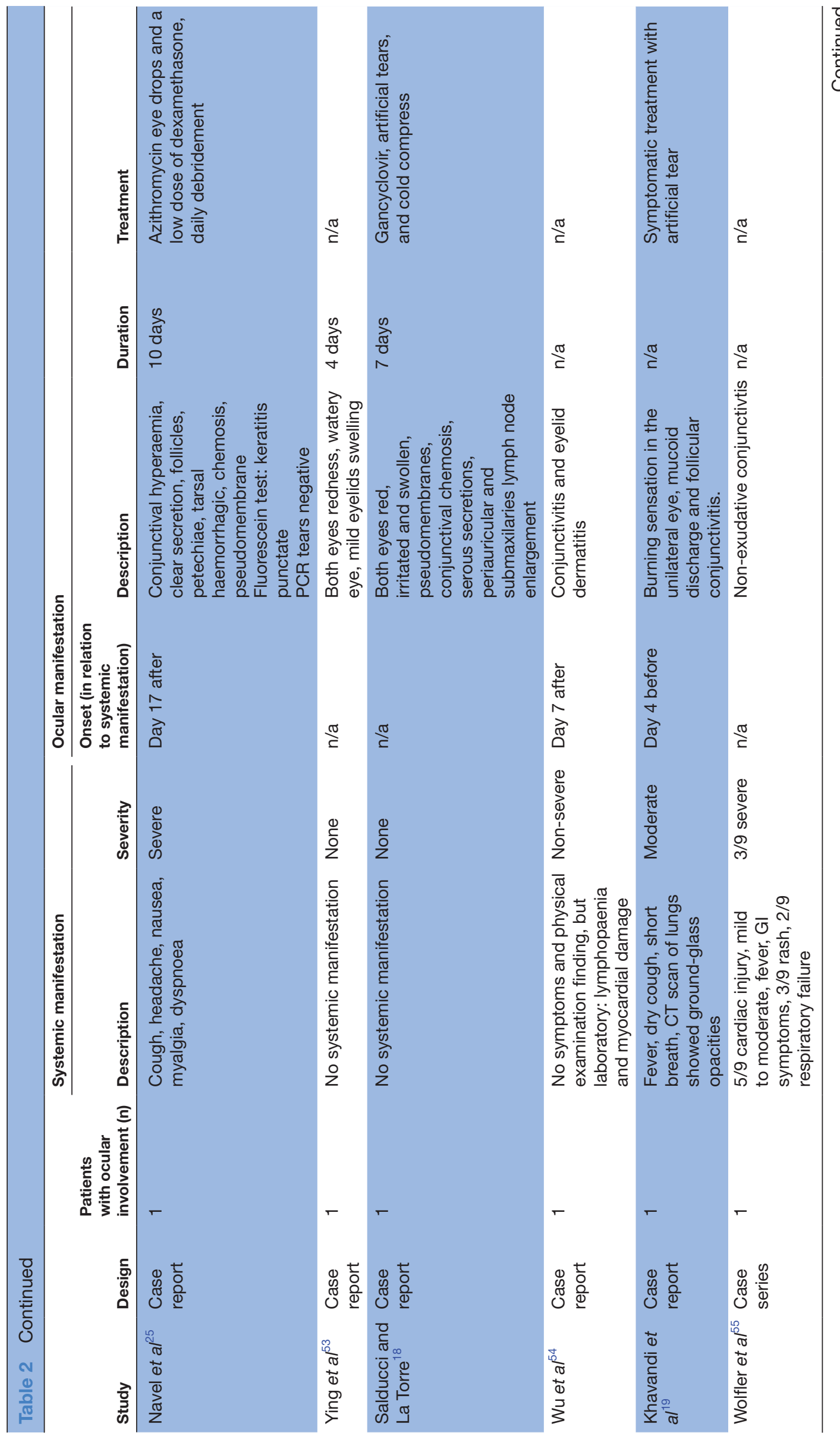




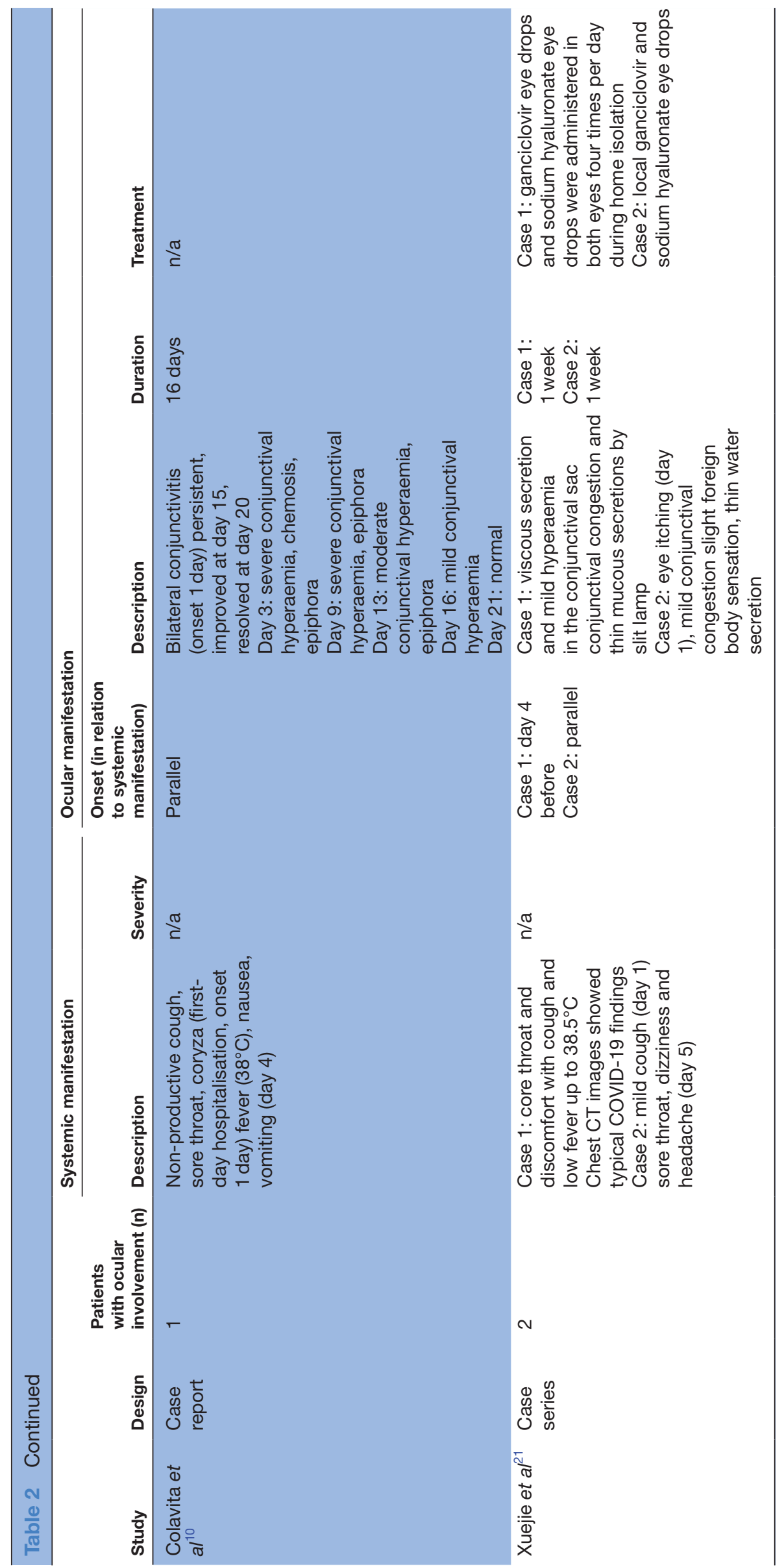




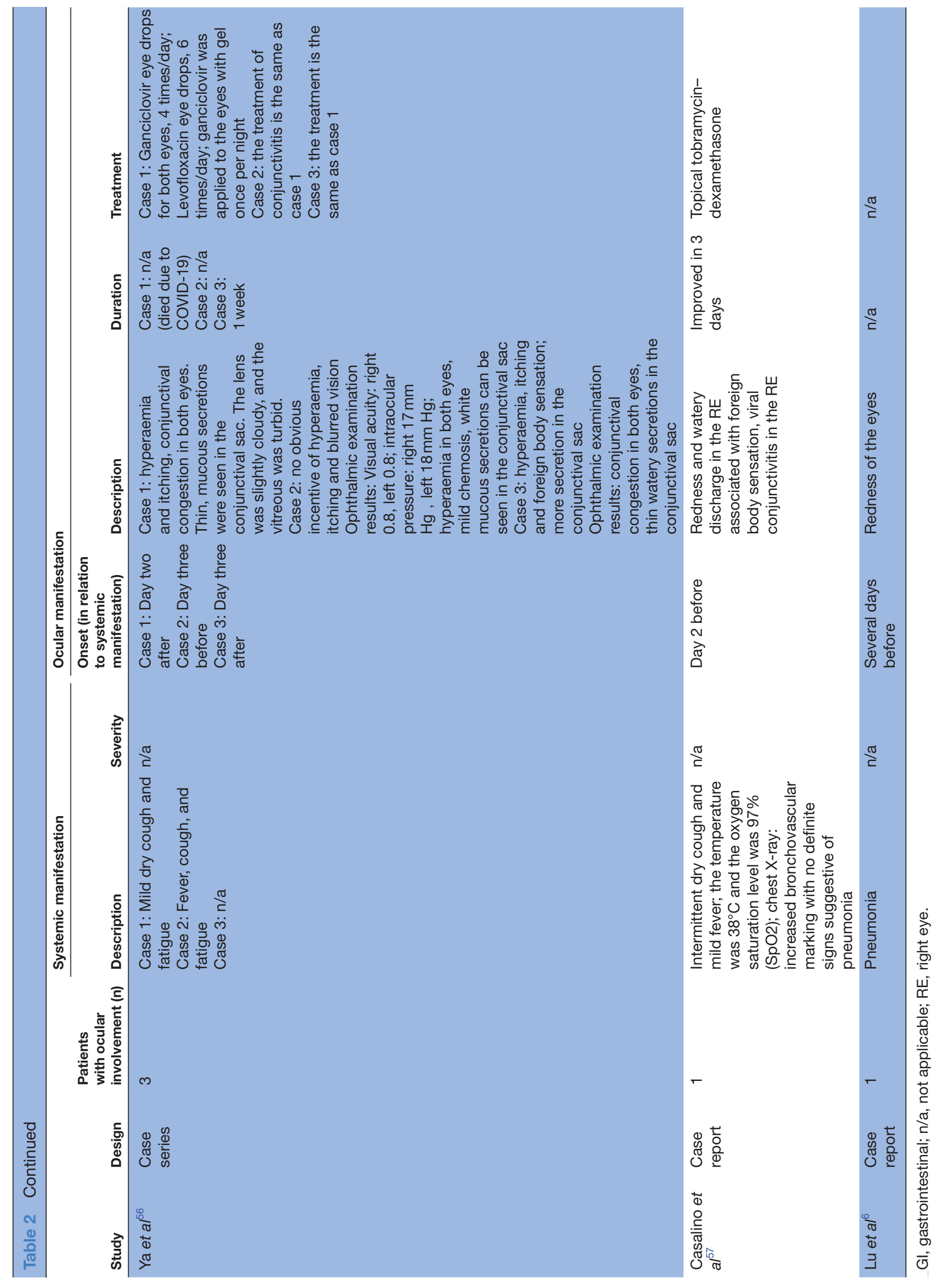




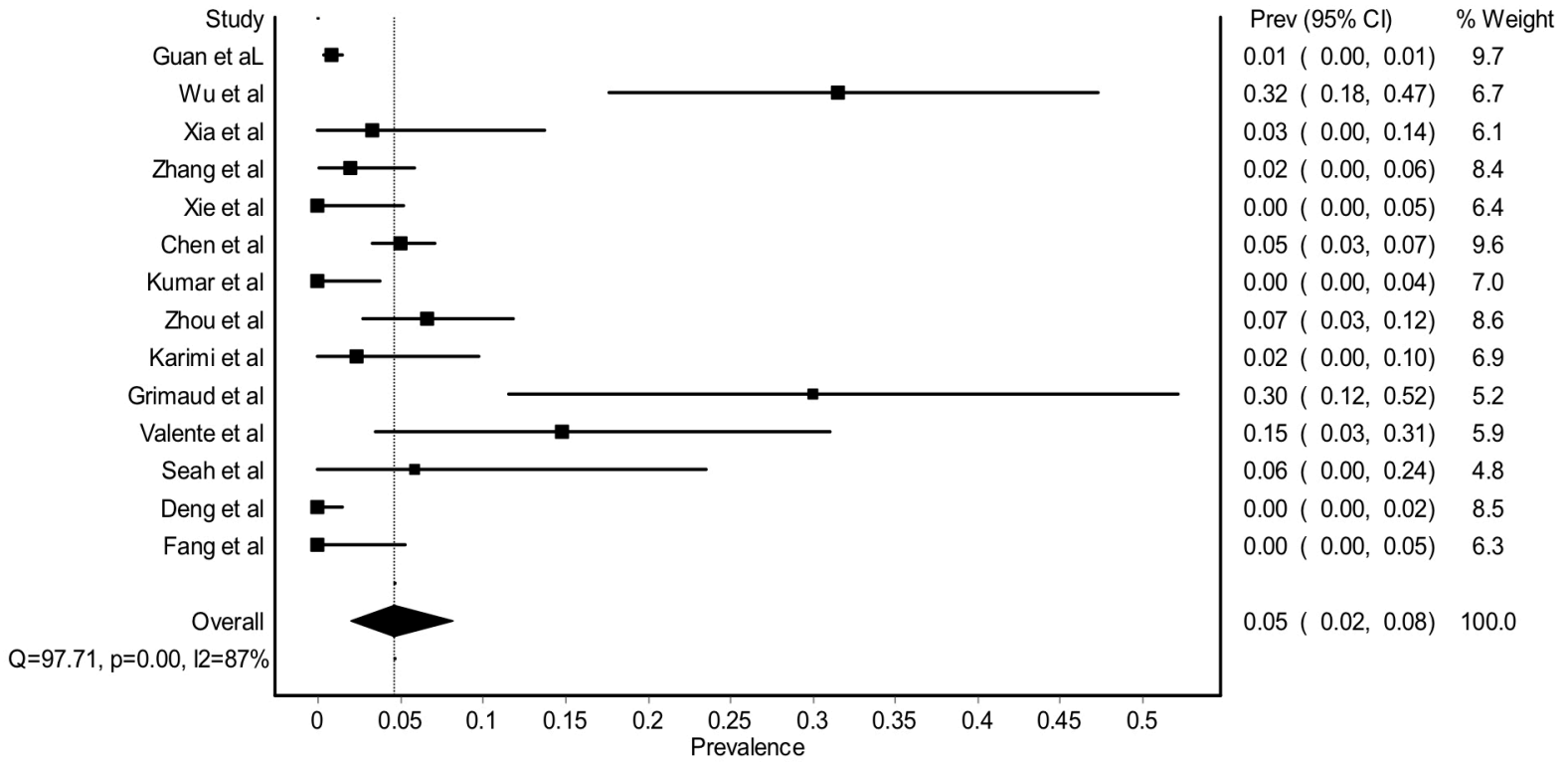

Figure 2 Forest plot estimating the pooled prevalence of ocular manifestation among COVID-19-related patients.

tears positivity from all COVID-19-related patients. If all available data of PCR positivity from tears were combined regardless of the presence of ocular manifestation, the pooled PCR positivity was only 0.04 (95\% CI $0.02 \%$ to 0.07 , figure 4$)$.

Relationship between the onset of ocular symptoms with the systemic manifestation of COVID-19

Several studies had reported the onset of ocular symptoms in relation to the presence of any systemic manifestation. From the pooled analysis, 0.28 (95\% CI $0.05 \%$ to 0.58 , figure 5) of ocular manifestation appeared without any systemic manifestations noticed by the patients. Frequent touching of the eyes with hands, ${ }^{16}$ elderly (age $>60$ years),${ }^{17-19}$ immunosuppression, ${ }^{17}$ lacrimal duct abnormalities, ${ }^{17}$ swimming ${ }^{20}$ and being healthcare workers $^{621}$ were reported to be possible predisposing factors for ocular manifestation of COVID-19.

Ocular manifestation can occur before, parallel or after the presence of systemic manifestation. Based on Scalinci et al $\mathrm{s}^{22}$ report, conjunctivitis could be the only manifestation of COVID-19 without any subsequent systemic manifestation. Interestingly, they found that the nasopharyngeal swab yielded a positive result in all of these patients. ${ }^{22}$ In another study, Cheema $e t a l^{20}$ found that when the ocular and systemic manifestation occurred at the same time in the early stage of the disease, PCR samples from both tears and nasopharyngeal mucosa could be positive. Moreover, they found that the cycle threshold for tears sample had a weaker signal compared with a nasopharyngeal swab. ${ }^{20}$ From the case series and case report studies, we found that the method and cycle threshold for PCR testing might influence the positivity rate of tear samples. Chen $e a^{23}$ and $\mathrm{Hu} e t a l^{17}$ took tear samples through putting sterile synthetic fibre or cotton swabs into the lower fornix of each eye with or without topical anaesthesia and found that this yielded a positive result, whereas Seah $e t a t^{24}$ took the tears samples with Schirmer strips and found that none of the samples was positive for SARS-CoV-2 even in the patient with conjunctivitis.

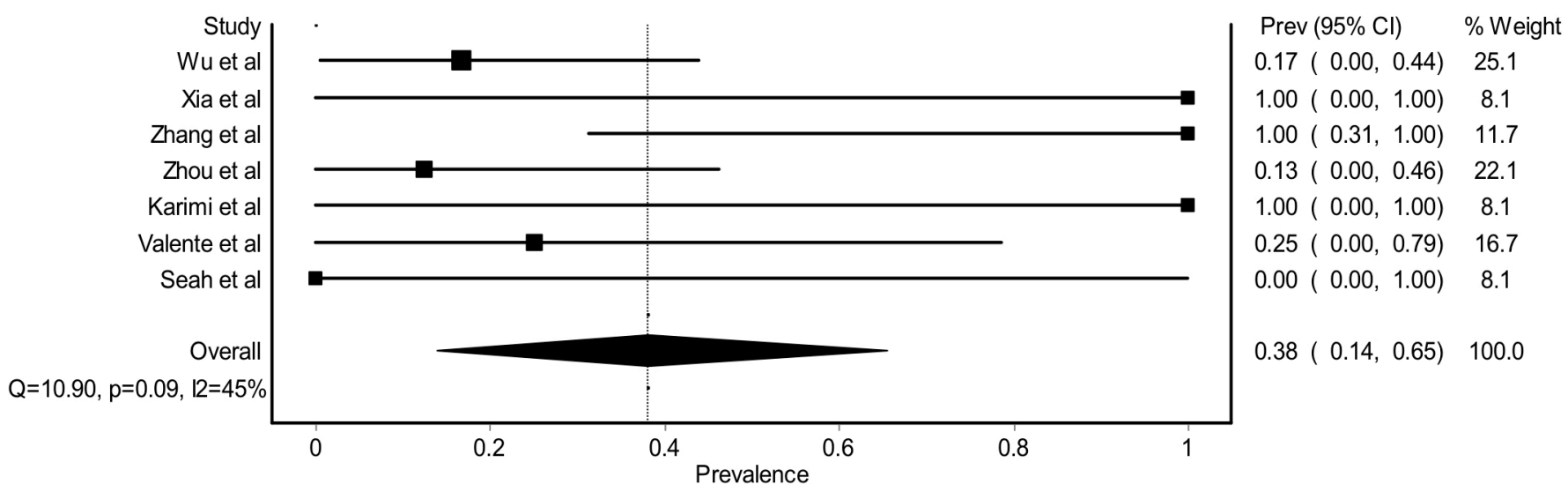

Figure 3 Forest plot estimating the pooled prevalence of PCR tears (+) among COVID-19-related patients with ocular manifestation. 


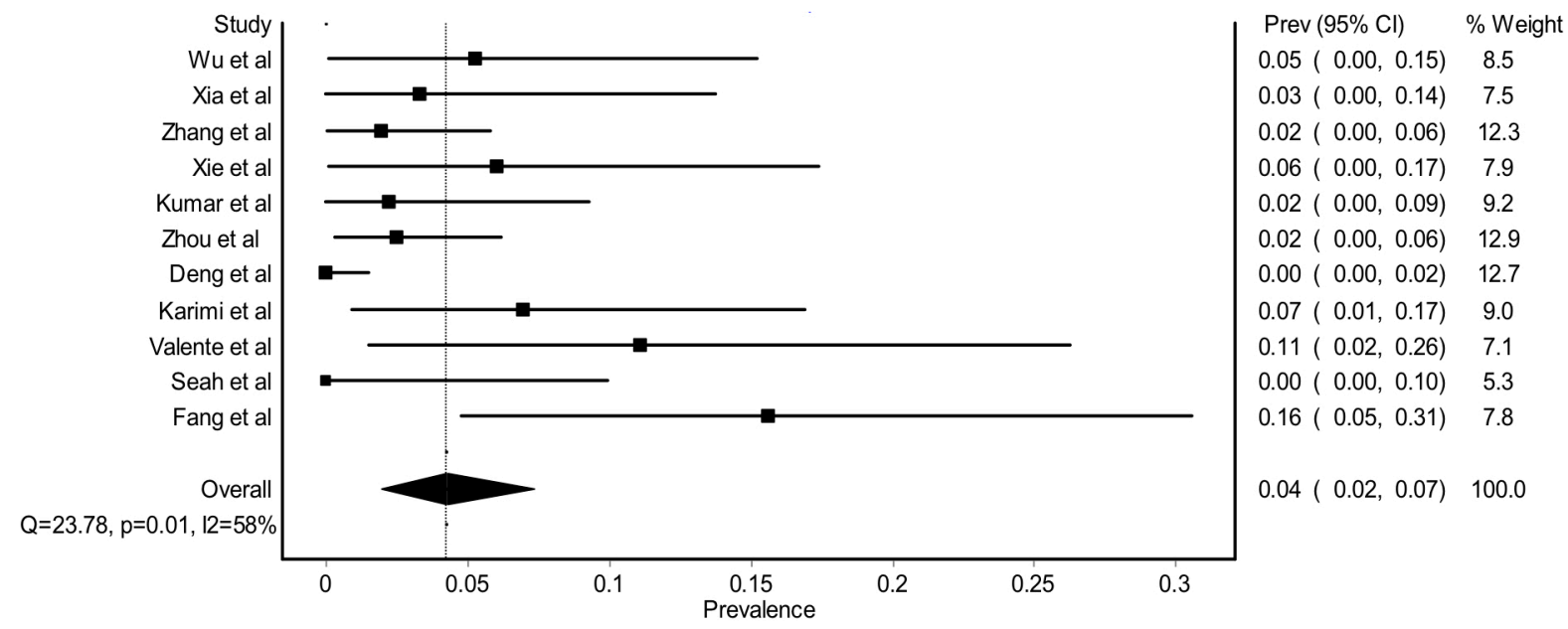

Figure 4 Forest plot estimating the pooled prevalence of PCR tears (+) among all COVID-19-related patients.

Severe COVID-19 was not associated with the presence of ocular manifestation (figure 6). A study by Navel et $a l^{25}$ found haemorrhagic conjunctivitis in an intubated patient with COVID-19 after several days of hospitalisation. However, PCR testing yield negative for SARS-CoV-2 even after excluding other potential microorganisms that could contribute to the ocular manifestation. ${ }^{25}$

\section{DISCUSSION}

Our systematic review and meta-analysis found a low prevalence of ocular manifestation in COVID-19-related patients. We also found that general PCR positivity from ocular samples was very low. However, when patients with COVID-19 had ocular manifestation, the positivity rate increased. The most common ocular manifestation being reported was epiphora, conjunctival injection and chemosis, similar to the other form of viral conjunctivitis with follicular reaction.

Our findings were in accordance with the previous systematic review by Aiello $e t a l^{26}$ that available earlier. Most of the studies included in their review were also included in our current study. In addition, we further performed a meta-analysis and elaborate on the association of ocular manifestation with the systemic manifestation of COVID19. Also, we added the data from case series and reports of individual patients with COVID-19 that explain the time course and disease severity of COVID-19 among those presenting with ocular manifestation.

The ocular surface can be the window of SARS-CoV-2 entry to the human body as the ACE-2 receptor found in conjunctiva and cornea. The TMPRSS 2 protease activity also appeared in the ocular surface. ${ }^{927}$ Recent work by Zhou $e t a l^{28}$ and Collin $e t a l^{29}$ found that ACE-2 receptor and TMPRSS 2 are highly found in the ocular surface epithelium, which can be the entry portal of coronavirus. Inflammation milieu could potentiate upregulation of ACE-2 and TMPRSS $2 .{ }^{29}$ As the virus may replicate after the ocular surface is being infected by the SARS-CoV-2, the virus can cause ocular manifestation prior to any systemic symptoms. Although most of the ocular manifestation being reported was associated with red eyes, a case report by $\mathrm{Hu}$ et $a l^{17}$ found that SARS-CoV-2 could be found in tears of asymptomatic eyes. In this case, further evaluation revealed a nasolacrimal duct obstruction. Even after the nasopharyngeal swab had turned negative, the tears were still found to be positive with low signal. ${ }^{17}$ Another report among paediatric patients with COVID-19 asymptomatic of ocular manifestation showed that their tears could also be positive for SARS-CoV-2. ${ }^{30}$ Moreover, it was noted that patients with only ocular manifestation could have nasopharyngeal mucosa positive for

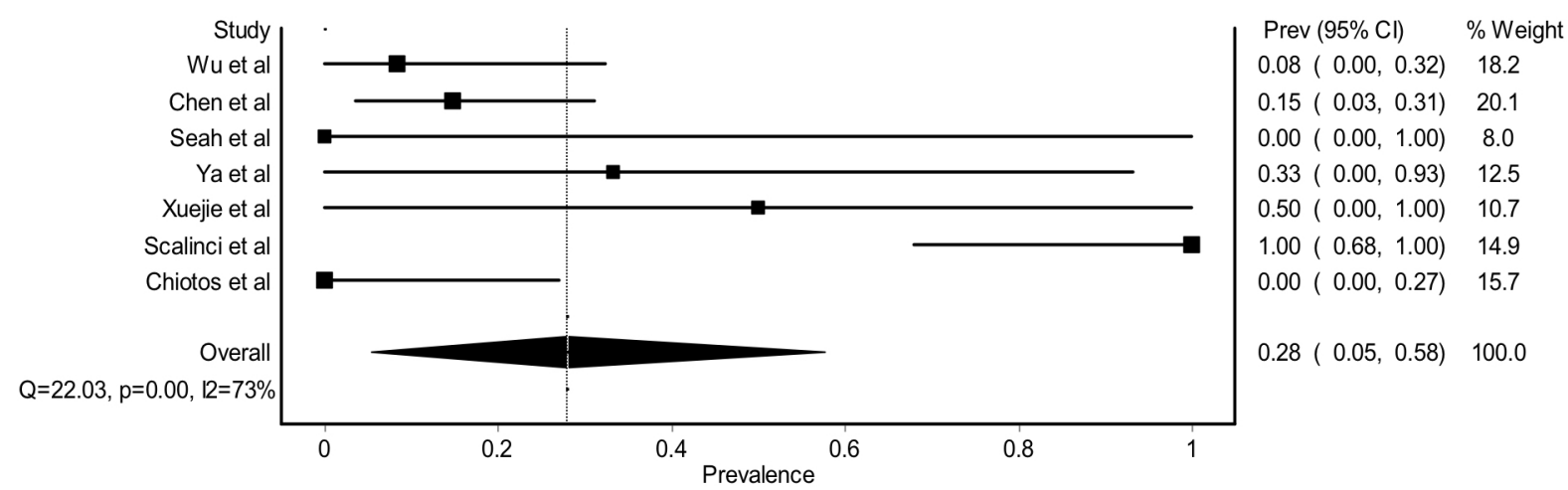

Figure 5 Forest plot estimating the pooled prevalence of ocular manifestation before any systemic manifestation among COVID-19 related patients with ocular manifestation. 


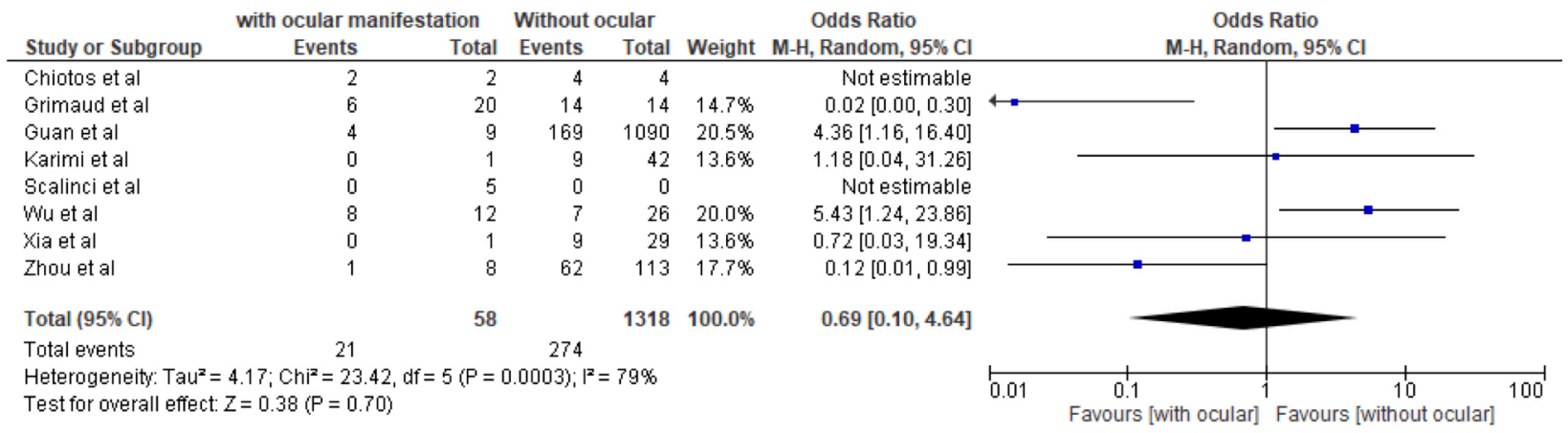

Figure 6 Forest plot estimating the OR of ocular manifestation in relation to severe COVID-19.

SARS-CoV-2. ${ }^{22}$ It raises the possibility that infection of COVID-19 in the eyes could lead to systemic manifestation via either nasolacrimal duct or touching the nose by hands contaminated with the virus from tears. Second, it may implicate that patients with ocular manifestation presenting with viral conjunctivitislike symptoms can be a source of transmission of SARS-CoV-2 to the population or unaware healthcare professionals. The possibility of the virus being transported to the nasopharynx was previously described, and it can occur experimentally in N95 respirator use without eye protection. ${ }^{1131}$

We found that the ocular manifestation was not associated with a severe form of COVID-19. However, subtle laboratory values may differ between patients with and without ocular manifestation. Two previous initial meta-analyses found the discrepancy of the evidence whether ocular manifestation could attribute to a severe form of COVID-19. ${ }^{32} 33$ Loffredo et $a l^{33}$ found that conjunctivitis among patients with COVID-19 was significantly associated with severe COVID-19. However, the conclusion was based only on three studies. As our study added more available data, we found the severity of COVID-19 was not associated with the ocular manifestation. From $\mathrm{Wu}$ et als $\mathrm{s}^{8}$ study, patients with COVID-19 presenting with ocular involvement had higher white blood cells, procalcitonin, $\mathrm{C}$ reactive protein and lactate dehydrogenase. Further evaluation needs to be done to confirm this finding. The severity of COVID-19 was probably mainly attributed to other systemic risk factors and the age of the patients.

Although we found that there was no significant ocular manifestation related to posterior segment abnormalities, the possible retinal involvement had been reported in patients with COVID-19. The ACE-2 receptor had also been reported in the human retina. ${ }^{34}$ Based on retinal biopsy specimens from 12 patients with COVID-19 who died, three specimens showed positive SARS-CoV-2 RNA with a weak signal. There was no report about any previous ocular manifestations until these patients died. However, the viral replication was not performed from these samples. ${ }^{35}$ Interestingly, Marinho et $a l^{36}$ reported recent ocular coherence tomography examination of COVID-19-related patients without any ocular manifestation. They found a subclinical change in ganglion cells and inner plexiform layers among these patients. ${ }^{36}$ Thus, ophthalmologists should be careful if they found this subtle change in OCT unintentionally or if they face patients with visual deterioration without typical of ocular surface manifestation of COVID-19-related patients.

Collecting adequate tears specimen for PCR testing may be a challenge in determining the true magnitude of ocular manifestation directly caused by SARS-CoV-2. We found a discrepancy in the result of PCR positivity of SARS-CoV-2 in tears by the methods of tear sampling. Seah $e t a l^{24}$ found Schirmer strip to obtain the virus and could not prove any positive result, even in patients with COVID-19 with conjunctivitis. Because of the high cycle threshold for detecting the presence of SARS-CoV-2 in tears compared with nasopharyngeal swab, ${ }^{10}$ the sampling method could potentially determine the positivity rate. Previously, Satpathy $e a^{37}$ and Ma et al ${ }^{38}$ found that scrapping specimen yielded a better PCR positivity rate compared with tears specimen based on the analysis of ocular herpes simplex cases. As described in a study evaluating nasal swab and aspirates, the quality of the samples for optimal virological diagnosis should be rich in cells. The more cells obtained, the more accurate the diagnosis. Even if there is no standardised minimum amount of cells for virological detection, this might apply in ocular samples for SARS-CoV-2 detection. ${ }^{39}$

Based on our findings, ophthalmologists should be aware of the possibility that patients may present only with viral conjunctivitis due to SARS-CoV-2 infection and may develop systemic manifestation several days later. Around one-third of patients who presented with ocular manifestation had signs and symptoms before they develop systemic COVID-19 manifestation. Moreover, around a third of COVID-19 related patients with ocular manifestations had SARS-CoV-2 PCR positive from the eye samples which could be infectious, although the infectivity of ocular samples yet to be determined. ${ }^{40}$ For hospitalised COVID-19 patient, any red eyes should be suspicious for the ocular manifestation of COVID-19 until proven otherwise. However, to our knowledge, there was no sight-threatening ocular condition found among 
COVID-19 patients that directly attributed to SARS-CoV-2 infection in the eyes. Moreover, the treatment option was varied across studies, ranging from artificial tears to antiviral eyedrops (tables 1 and 2). The ocular manifestation could improve in all studies without any complication. Urgent consultation with an ophthalmologist may not be needed as long as the ocular manifestation does not get worse during the treatment course. For the ophthalmologist facing viral conjunctivitis-like patients without any systemic manifestation but high risk of being infected with SARS-CoV-2, it is reasonable if the tears specimen for PCR testing could be obtained in a repeated manner or in combination with the nasopharyngeal swab. Performing routine PCR testing for SARS-CoV-2 among patients with COVID-19 without ocular manifestation may yield a very low positivity rate, as previously reported by Deng et al. ${ }^{41}$ Eye protection and mask should be worn in managing patients with red eyes in daily clinical practice. A recent systematic review found that using eye protection or face mask, in addition to physical distancing and using respirators, could further reduce the risk of being infected by SARS-CoV-2. ${ }^{42}$

This study had several limitations. First, the general positivity rate of ocular samples for SARS-CoV-2 estimated mostly by studies conducting in hospital settings. This value can be overestimated. Second, the description of onset and ocular manifestation varies between studies included. Also, most of the studies did not describe in detail how to obtain tear samples. As we previously mentioned, the technique possibly influences the positivity rate. Subgroup analysis of pooled prevalence could not be performed further as the data for ocular manifestations were limited. Language bias may also be of relevance in this systematic review.

\section{CONCLUSIONS}

After carefully reviewing the available literature, we found that ocular manifestation can be the first presenting symptom of COVID-19. Eye infection of SARS-CoV-2 could potentially lead to systemic manifestation, although its evidence needs further investigation. Patients with COVID-19 with ocular manifestation were not associated with severe COVID-19. Detecting SARS-CoV-2 from tears yielded a relatively low positivity rate. However, we acknowledged the possibility of SARS-CoV-2 transmission from patients with COVID-19 presenting with only ocular manifestation.

\section{Contributors All authors contributed significantly to this study.}

Funding The authors have not declared a specific grant for this research from any funding agency in the public, commercial or not-for-profit sectors.

Competing interests None declared.

Patient and public involvement Patients and/or the public were not involved in the design, conduct, reporting or dissemination plans of this research.

Patient consent for publication Not required.

Provenance and peer review Not commissioned; externally peer reviewed.

Data availability statement All data relevant to the study are included in the article.
Open access This is an open access article distributed in accordance with the Creative Commons Attribution Non Commercial (CC BY-NC 4.0) license, which permits others to distribute, remix, adapt, build upon this work non-commercially, and license their derivative works on different terms, provided the original work is properly cited, appropriate credit is given, any changes made indicated, and the use is non-commercial. See: http://creativecommons.org/licenses/by-nc/4.0/.

ORCID iD

Rina La Distia Nora http://orcid.org/0000-0002-7026-5341

\section{REFERENCES}

1 Pascarella G, Strumia A, Piliego C, et al. COVID-19 diagnosis and management: a comprehensive review. J Intern Med 2020;288:192-206.

2 Singhal T. A review of coronavirus Disease-2019 (COVID-19). Indian $J$ Pediatr 2020;87:281-6.

3 World Health Organization. Coronavirus disease 2019 (COVID-19) situation report-115; 2020. https://apps.who.int/iris/bitstream/ handle/10665/332090/nCoVsitrep14May2020-eng.pdf?sequence= 1\&isAllowed=y [Accessed 06/17/2020].

4 World Health Organization. Infection prevention and control during health care when COVID-19 is suspected; 2020. https://www. who.int/publications-detail-redirect/10665-331495 [Accessed 06/17/2020].

5 Loon S-C, Teoh SCB, Oon LLE, et al. The severe acute respiratory syndrome coronavirus in tears. Br J Ophthalmol 2004;88:861-3.

6 C-W L, Liu X-F, Jia Z-F. 2019-nCoV transmission through the ocular surface must not be ignored. Lancet 2020:e39.

7 Guan W-J, Ni Z-Y, Hu Y, et al. Clinical characteristics of coronavirus disease 2019 in China. N Engl J Med 2020;382:1708-20.

8 Wu P, Duan F, Luo C, et al. Characteristics of ocular findings of patients with coronavirus disease 2019 (COVID-19) in Hubei Province, China. JAMA Ophthalmol 2020;138:575-8.

9 Hui KPY, Cheung M-C, Perera RAPM, et al. Tropism, replication competence, and innate immune responses of the coronavirus SARS-CoV-2 in human respiratory tract and conjunctiva: an analysis in ex-vivo and in-vitro cultures. Lancet Respir Med 2020;8:687-95.

10 Colavita F, Lapa D, Carletti F, et al. SARS-CoV-2 isolation from ocular secretions of a patient with COVID-19 in Italy with prolonged viral RNA detection. Ann Intern Med 2020;173:242-3.

11 Belser JA, Rota PA, Tumpey TM. Ocular tropism of respiratory viruses. Microbiol Mol Biol Rev 2013;77:144-56.

12 Moher D, Shamseer L, Clarke M, et al. Preferred reporting items for systematic review and meta-analysis protocols (PRISMA-P) 2015 statement. Syst Rev 2015;4:1.

13 Wells G, Shea B, O'Connel D, et al. The Newcastle-Ottawa Scale (NOS) for assessing the quality of nonrandomised studies in metaanalyses [Internet] 2019.

14 Murad MH, Sultan S, Haffar S, et al. Methodological quality and synthesis of case series and case reports. BMJ Evid Based Med 2018;23:60-3.

15 Zhang X, Chen X, Chen L, et al. The evidence of SARS-CoV-2 infection on ocular surface. Ocul Surf 2020;18:360-2.

16 Hong N, Yu W, Xia J, et al. Evaluation of ocular symptoms and tropism of SARS-CoV-2 in patients confirmed with COVID-19. Acta Ophthalmol 2020. doi:10.1111/aos.14445. [Epub ahead of print: 26 Apr 2020].

17 Hu Y, Chen T, Liu M, et al. Positive detection of SARS-CoV-2 combined HSV1 and HHV6B virus nucleic acid in tear and conjunctival secretions of a non-conjunctivitis COVID-19 patient with obstruction of common lacrimal duct. Acta Ophthalmol 2020. doi:10.1111/aos.14456. [Epub ahead of print: 14 May 2020].

18 Salducci M, La Torre G. COVID-19 emergency in the cruise's ship: a case report of conjunctivitis. Clin Ter 2020;171:e189-91.

19 Khavandi S, Tabibzadeh E, Naderan M, et al. Corona virus disease-19 (COVID-19) presenting as conjunctivitis: atypically highrisk during a pandemic. Cont Lens Anterior Eye 2020;43:211-2.

20 Cheema M, Aghazadeh $\mathrm{H}$, Nazarali S, et al. Keratoconjunctivitis as the initial medical presentation of the novel coronavirus disease 2019 (COVID-19). Can J Ophthalmol 2020;2.

21 Xuejie L, Ming W, Jing D, et al. Novel coronavirus disease with conjunctivitis and conjunctivitis as first symptom: two cases report. CJEO 2020.

22 Scalinci SZ, Trovato Battagliola E. Conjunctivitis can be the only presenting sign and symptom of COVID-19. IDCases 2020;20:e00774.

23 Chen L, Liu M, Zhang Z, et al. Ocular manifestations of a hospitalised patient with confirmed 2019 novel coronavirus disease. $\mathrm{Br} J$ Ophthalmol 2020;104:748-51. 
24 Seah IYJ, Anderson DE, Kang AEZ, et al. Assessing viral shedding and infectivity of tears in coronavirus disease 2019 (COVID-19) patients. Ophthalmology 2020;127:977-9.

25 Navel V, Chiambaretta F, Dutheil F. Haemorrhagic conjunctivitis with pseudomembranous related to SARS-CoV-2. Am J Ophthalmol Case Rep 2020;19:100735.

26 Aiello F, Gallo Afflitto G, Mancino R, et al. Coronavirus disease 2019 (SARS-CoV-2) and colonization of ocular tissues and secretions: a systematic review. Eye 2020;34:1206-11.

27 Sungnak W, Huang N, Bécavin C, et al. SARS-CoV-2 entry factors are highly expressed in nasal epithelial cells together with innate immune genes. Nat Med 2020;26:681-7.

28 Zhou L, Xu Z, Castiglione GM, et al. ACE2 and TMPRSS2 are expressed on the human ocular surface, suggesting susceptibility to SARS-CoV-2 infection. Ocul Surf 2020;18:537-44.

29 Collin J, Queen R, Zerti D, et al. Co-expression of SARS-CoV-2 entry genes in the superficial adult human conjunctival, limbal and corneal epithelium suggests an additional route of entry via the ocular surface. Ocul Surf 2020. doi:10.1016/j.jtos.2020.05.013. [Epub ahead of print: 03 Jun 2020].

30 Valente P, larossi G, Federici M, et al. Ocular manifestations and viral shedding in tears of pediatric patients with coronavirus disease 2019: a preliminary report. J Aapos 2020. doi:10.1016/j. jaapos.2020.05.002. [Epub ahead of print: 09 Jun 2020].

31 Bischoff WE, Reid T, Russell GB, et al. Transocular entry of seasonal influenza-attenuated virus aerosols and the efficacy of n95 respirators, surgical masks, and eye protection in humans. $J$ Infect Dis 2011;204:193-9.

32 Liu M, Dai C, Lv X, et al. Letter to the editor: are severe COVID-19 patients more susceptible to conjunctivitis? J Med Virol 2020. doi:10.1002/jmv.26084. [Epub ahead of print: 29 May 2020].

33 Loffredo L, Pacella F, Pacella E, et al. Conjunctivitis and COVID-19: a meta-analysis. J Med Virol 2020. doi:10.1002/jmv.25938. [Epub ahead of print: 24 Apr 2020].

34 Senanayake PdeS, Drazba J, Shadrach K, et al. Angiotensin II and its receptor subtypes in the human retina. Invest Ophthalmol Vis Sci 2007;48:3301-11.

35 Casagrande M, Fitzek A, Püschel K, et al. Detection of SARS-CoV-2 in human retinal biopsies of deceased COVID-19 patients. Ocul Immunol Inflamm 2020;28:721-5.

36 Marinho PM, Marcos AAA, Romano AC, et al. Retinal findings in patients with COVID-19. Lancet 2020;395:1610.

37 Satpathy G, Mishra AK, Tandon R, et al. Evaluation of tear samples for herpes simplex virus 1 (HSV) detection in suspected cases of viral keratitis using PCR assay and conventional laboratory diagnostic tools. Br J Ophthalmol 2011;95:415-8.

38 Ma J-X, Wang L-N, Zhou R-X, et al. Real-time polymerase chain reaction for the diagnosis of necrotizing herpes stromal keratitis. Int J Ophthalmol 2016;9:682-6.

39 Bonnin P, Miszczak F, Kin N, et al. Study and interest of cellular load in respiratory samples for the optimization of molecular virological diagnosis in clinical practice. BMC Infect Dis 2016;16:384.
40 Sun C-B, Wang Y-Y, Liu G-H, et al. Role of the eye in transmitting human coronavirus: what we know and what we do not know. Front Public Health 2020;8:155.

41 Deng $\mathrm{C}$, Yang $\mathrm{Y}$, Chen $\mathrm{H}$, et al. Low risk of SARS-CoV-2 transmission through the ocular surface. Acta Ophthalmol 2020;395.

42 Chu DK, Akl EA, Duda S, et al. Physical distancing, face masks, and eye protection to prevent person-to-person transmission of SARS-CoV-2 and COVID-19: a systematic review and meta-analysis. Lancet 2020;395:1973-87.

43 Xia J, Tong J, Liu M, et al. Evaluation of coronavirus in tears and conjunctival secretions of patients with SARS-CoV-2 infection. $J$ Med Virol 2020;92:589-94.

44 Xie H-T, Jiang S-Y, Xu K-K, et al. SARS-CoV-2 in the ocular surface of COVID-19 patients. Eye Vis 2020;7:23.

45 Chen L, Deng C, Chen X, et al. Ocular manifestations and clinical characteristics of 535 cases of COVID-19 in Wuhan, China: a crosssectional study. Acta Ophthalmol 2020. doi:10.1111/aos.14472. [Epub ahead of print: 18 May 2020].

46 Kumar K, Prakash AA, Gangasagara SB, et al. Presence of viral RNA of SARS-CoV-2 in conjunctival swab specimens of COVID-19 patients. Indian J Ophthalmol 2020;68:1015-7.

47 Zhou Y, Duan C, Zeng Y, et al. Ocular findings and proportion with conjunctival SARS-COV-2 in COVID-19 patients. Ophthalmology 2020;127:982-3.

48 Karimi S, Arabi A, Shahraki T, et al. Detection of severe acute respiratory syndrome Coronavirus-2 in the tears of patients with coronavirus disease 2019. Eye 2020;34:1220-3.

49 Grimaud M, Starck J, Levy M, et al. Acute myocarditis and multisystem inflammatory emerging disease following SARS-CoV-2 infection in critically ill children. Ann Intensive Care 2020;10:69.

50 Fang Z, Zhang Y, Hang C, et al. Comparisons of viral shedding time of SARS-CoV-2 of different samples in ICU and non-ICU patients. $J$ Infect 2020;81:147-78.

51 Daruich A, Martin D, Bremond-Gignac D. Ocular manifestation as first sign of coronavirus disease 2019 (COVID-19): interest of telemedicine during the pandemic context. J Fr Ophtalmol 2020;43:389-91.

52 Chiotos K, Bassiri H, Behrens EM, et al. Multisystem inflammatory syndrome in children during the coronavirus 2019 pandemic: a case series. J Pediatric Infect Dis Soc 2020;9:393-8.

53 Ying NY, Idris NS, Muhamad R, et al. Coronavirus disease 2019 presenting as conjunctivitis. Korean J Fam Med 2020. doi:10.4082/ kifm.20.0090. [Epub ahead of print: 01 Jun 2020].

54 Wu P, Liang L, Chen C, et al. A child confirmed COVID-19 with only symptoms of conjunctivitis and eyelid dermatitis. Graefes Arch Clin Exp Ophthalmol 2020;258:1565-6.

55 Wolfler A, Mannarino S, Giacomet V, et al. Acute myocardial injury: a novel clinical pattern in children with COVID-19. Lancet Child Adolesc Health 2020;4:e26-7.

56 Ya Y, Yanping S, Ming Y, et al. Novel coronavirus pneumonia combined with conjunctivitis: three cases report. CJEO 2020.

57 Casalino G, Monaco G, Di Sarro PP, et al. Coronavirus disease 2019 presenting with conjunctivitis as the first symptom. Eye 2020;34:1235-6. 